Formal Methods in Systems Engineering 


\section{Formal Methods in Systems Engineering}

Edited by Peter Ryan and Chris Sennett

Springer-Verlag

London Berlin Heidelberg New York Paris Tokyo Hong Kong Barcelona Budapest 
Peter Ryan, BSc, PhD

Chris Sennett, MA, DPhil

Defence Research Agency

St Andrew's Road, Malvern

Worcestershire WR14 3PS, UK

ISBN-13:978-3-540-19751-5

e-ISBN-13:978-1-4471-1975-3

DOI: $10.1007 / 978-1-4471-1975-3$

Library of Congress Cataloging-in-Publication Data applied for

Apart from any fair dealing for the purposes of research or private study, or criticism or review, as permitted under the Copyright, Designs and

Patents Act 1988, this publication may only be reproduced, stored or transmitted, in any form, or by any means, with the prior permission in writing of the publishers, or in the case of reprographic reproduction in accordance with the terms of licences issued by the Copyright Licensing Agency. Enquiries concerning reproduction outside those terms should be sent to the publishers.

c) Springer-Verlag London Limited 1993 except as indicated.

The use of registered names, trademarks etc. in this publication does not imply, even in the absence of a specific statement, that such names are exempt from the relevant laws and regulations and therefore free for general use.

The publisher makes no representation, express or implied, with regard to the accuracy of the information contained in this book and cannot accept any legal responsibility or liability for any errors or omissions that may be made.

Typesetting: Camera ready by editors

34/3830-543210 Printed on acid-free paper 


\section{Preface}

Digital systems are becoming ever more pervasive in our modern, technological society. More and more aspects of our lives rely increasingly heavily on such systems. Many of these applications are of great complexity and criticality. This criticality may be of a safety nature such as "fly-by-wire" avionics and nuclear power station control systems, or of a security nature such as financial transaction systems and databases of sensitive information. In any case it is of great importance that these systems perform correctly and reliably.

Set against this is the fact that digital systems engineering has not come of age. It is in some ways better thought of as being still in an "arts and crafts" stage rather than as a true engineering or scientific discipline. Nevertheless, systems engineering is rising to meet the challenge of mastering the complexity and providing suitably high levels of assurance of correct and reliable behaviour for such critical applications.

Over the last few decades significant strides have been made in the theoretical foundations of the subject but there are still many open questions. Methods and tools such as structured methods and compilers have also developed. These improve the situation but do not solve the underlying problem.

A comparatively new line of attack comes under the name of "formal methods". In some ways this is perhaps a slightly unfortunate and misleading title but is probably here to stay. It is unfortunate in that it suggests the essence of the approach is formality, that is, comprising elaborate, abstruse symbol manipulations. Certainly such symbol-pushing exercises can play a role but they are by no means the totality of the approach, as the ensuing pages will demonstrate.

Formal methods can be roughly defined as the application of mathematical techniques to the specification and development of digital systems. Such techniques include the use of a mathematical notation to increase the precision of specifications, the use of abstraction and modularity to reduce a problem to "mind-sized" chunks, and the use of analytic techniques to establish that a design or specification has certain properties. More precise definitions can be found in the text, notably in Joseph Goguen's Introduction.

The underlining thesis of this book is the belief that formal methods can make an impact on these problems. They should also help to produce better quality products in more reliable timescales and budgets. By using the more advanced proof and refinement techniques, they can be used to produce systems with high assurance of "correct" behaviour (although this will tend to have a trade-off in terms of production timescales and cost). It must however be acknowledged that on the whole formal methods have yet to make a significant impact on "real", 
industrial-scale systems engineering. It is an important question, with both technical and social ramifications, as to why this is the case.

In the light of this a workshop (FM'89) was organised and held in July 1989 in Halifax, Nova Scotia to try to assess the state of the art in formal methods and make recommendations for future research and development in the field. The workshop was sponsored by the governments of Canada, the UK and the US and drew together some 50 experts in the field from academia, industry and government in the 3 countries.

One of the key conclusions to emerge from this workshop was that in order to facilitate the transfer of the technology and make formal methods effective in industrial applications it would be necessary to understand how to integrate them with existing software engineering practice, such as quality assurance, structured design, testing and so on. There is a common perception of formal methods as all-or-nothing approaches which exclude other techniques. In fact they can and should co-exist in a fruitful symbiosis with existing practice, and are best viewed as another weapon in the engineer's armoury to achieve quality, dependable systems.

This was chosen as the theme of a follow-on workshop held in September 1991 in Drymen, Scotland with a similar, and partly overlapping, group of participants. This book documents the outcome of the workshop and is intended to give a perspective on the state of the art with regard to the practical use of formal methods in industry. Much of the material was presented as invited talks at the workshop and developed subsequently. Also included are reports from three parallel working groups held during the workshop. These present, as far as can be attained, the consensus of the practitioners on the use of formal methods under the headings:

- Modelling

- Quality assurance

- Design methods

The first of these was aimed at understanding the role of mathematical modelling techniques in specification and design. The second was to study how formal methods could interact with and complement existing quality assurance techniques. The last was similarly intended to investigate the integration of formal methods into existing design methods.

To complete the appreciation of the state of the art, a survey of formal methods tools and applications was conducted throughout 1992 and the results of this are given in the appendix.

After the Introduction by Joseph Goguen chapters 2 and 3 deal with some social aspects of formal methods. Margaret Tierney examines the role of formal methods and the way they are commonly perceived. In particular she discusses the way they 
have tended to be associated with the high-criticality, defencetype applications. In the third chapter, Donald MacKenzie examines the idea of "proof", in particular formal proof, and shows that it is far from being as cut-and-dried as is commonly thought.

Three further chapters address approaches to the problem of developing large systems. The first of these is by Jean-Raymond Abrial. He is the driving force behind the development of the Btechnologies. This is a unified and elegant approach, comprising a notation, method and supporting tools. In this chapter he outlines the ideas behind this approach and some of the lessons learnt. The next chapter is by Pamela Zave. This describes research done with Michael Jackson on techniques for the composition of specifications. By using such techniques, a problem may be broken down into manageable pieces which can then be combined in a controlled fashion. The third is by John Wordsworth, and addresses the often rather neglected issue of ways in which formal methods can be used to improve documentation throughout the development life cycle.

The remaining chapters document various experiences gained in the course of the application of certain formal methods to "real" applications. Anthony Hall draws on his experience in the design and development of an air traffic control system for the CAA. In particular he addresses the issue of what constitutes a "good" specification. Finally Victor Basili examines the nature of "quality", emphasising that QA should be process-oriented rather than product-oriented.

The FM workshops were unusual in the way that they deliberately drew together key players from industry, government and academia. In this way the providers, funders and consumers of the technology all had an opportunity to meet and discuss their hopes and concerns. We hope that this book will make a contribution to the awareness of the potential (and limitations) of an important new technology.

February 1993

Peter Ryan

Chris Sennett

\section{Acknowledgements}

We should like to thank the CSE in Canada, CESG in the UK and NSA in the States for sponsoring the workshop on which this material is based.

Thanks to Stephanie Smit of Elsevier for permission to reproduce the contribution from Jean-Raymond Abrial.

Thanks to Mark Hewitt, Colin O'Halloran and Paul Rathbone for help with the $\mathrm{L}^{\mathrm{A}} \mathrm{T}_{\mathrm{E}} \mathrm{X}$ typesetting. 


\section{Contents}

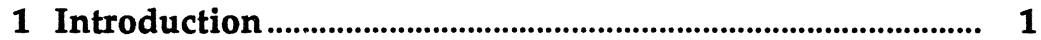

1.1 What Are Formal Methods? .......................................... 1

1.2 Formal Methods and Mathematics .................................. 2

1.3 What Good Are Formal Methods? ................................... 2

1.4 The Myth of Control ........................................................... 4

1.5 Hyperprogramming............................................................ 5

1.6 Recommendations ................................................................ 6

2 Formal Methods of Software Development: Painted into the Corner of High-Integrity Computing? ................ 11

2.1 The Dominant Rationale for Formal Methods ............ 12

2.2 Some Pragmatic Objections to Formal Methods........ 13

2.3 Dissolving Resistance to Formal Methods? ................. 15

2.4 A Brief Sketch of Formal Methods in High-Integrity Computing ............................................... 16

2.5 The Projects....................................................................... 16

2.6 The Formal Methods Community in High-Integrity Computing ............................................. 18

2.6.1 The Clients.............................................................. 18

2.6.2 The Developers......................................................... 18

2.7 The Information Networks ........................................... 19

2.8 Does the Use of Formal Methods Within High-Integrity Computing Perpetuate its "Myths"?

3 The Social Negotiation of Proof: An Analysis and a

Further Prediction

3.1 Background ……......................................................... 23

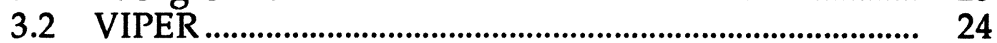

3.3 Disputing "Proof" ........................................................... 25

3.4 Formal Proof and Rigorous Argument ......................... 27

3.5 A Further Prediction ...................................................... 28

3.6 Conclusion ................................................................. 29

4 On Constructing Large Software Systems.......................... 32

4.1 Introduction .................................................................. 32

4.2 People .................................................................................. 32

4.3 Frames .................................................................... 33

4.4 Sets........................................................................... 35

4.5 Programs .............................................................. 36

4.6 Proof ............................................................................ 38

4.7 Tools ......................................................................... 38

4.8 Conclusion …….......................................................... 39

4.9 Acknowledgments ........................................................ 40

5 Composition of Descriptions: A Progress Report ............ 41

5.1 Introduction ...................................................................... 41 
5.2 Why Compose Descriptions ? ......................................... 41

5.3 What is Described? ...................................................... 43

5.4 What is a Description? ................................................... 45

5.5 What is Composition? ................................................... 47

5.6 Description Reuse ....................................................... 48

5.7 Conclusion ...................................................................... 48

6 Integrating Methods in Practice ......................................... 51

6.1 Introduction .............................................................. 51

6.2 Development and Development Methods .................... 52

6.3 Aspects of Specification .................................................... 54

6.4 Aspects of Design ............................................................... 56

6.4.1 Detail and Structure ........................................... 56

6.4.2 Reification and Algorithm Design ..................... 58

6.4.3 Summary ................................................................ 58

6.5 Implications for Research and Development ............. 59

7 Formal Methods and Product Documentation .................. 60

7.1 Introduction ...................................................................... 60

7.2 The Fully Formalised Software Product ....................... 60

7.3 The Elements of Product Documentation .................... 60

7.4 Product Documentation and the Product Range....... 65

7.5 Product Documentation and Product Development .................................................................... 65

7.6 Product Documentation and Customers ..................... 66

7.7 Developing from a Fully Formalised Base.................. 67

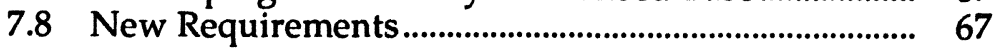

7.9 Errors Detected Before Release ...................................... 68

7.10 Errors Detected After Release ....................................... 68

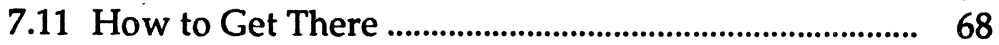

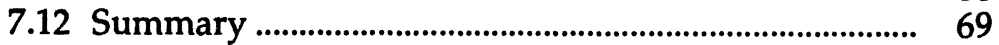

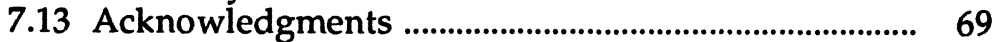

8 Software Quality: A Modelling and Measurement

View

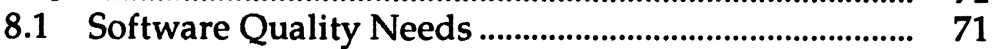

8.2 Modelling Software Experiences .................................... 72

8.3 Model Evolution .............................................................. 75

8.4 An Organization for Packaging Experience Models for Reuse .......................................................................... 78

8.5 Conclusions ................................................................ 79

9 Modelling Working Group Summary …………................... 81

9.1 Description ............................................................................. 81

9.2 Discussion Topics ................................................................. 81

9.3 Discussion .................................................................... 82

9.3.1 Why and to What End Formal Methods? ......... 83

9.3.2 What is a Formal Method? .................................. 83

9.4 How Does Mathematical Modelling Help to Increase our Understanding of Digital Systems? ...... 84 
9.4.1 What Are the Limits of Mathematical Modelling?

9.5 What is Required to Validate that a

Mathematical Model Describes a Digital

System Accurately?

9.5.1 What Insights do Other Fields of

Engineering Provide?

9.5.2 What Can we Do by 1995 with the Mathematical Modelling Capabilities we Have Now?

9.5.3 What Is our Target for the Year 2000?

9.5.4 Formal Methods and Safety Standards

9.6 Conclusions

10 Quality Assurance Working Group 86

10.1 Group Description.

10.2 Quality Assurance vs. Quality Control

10.3 What Is a Formal Method?

10.4 Integration of Formal Methods and Quality Control

10.5 A Plan to Integrate Formal Methods into QC/QA

10.5.1 Phase 1

10.5.2 Phase 2

10.5.3 Phase 3

11 Design Methods Working Group

11.1 Description

11.2 The Context of Formal Methods

11.3 The Role of Proof in Assurance.

11.4 Analytical Capabilities of Formal Methods

11.5 Foundational Capabilities of Formal Methods

11.6 The Role of Formal Methods with Respect to the Software Development Process

11.7 Formal Methods During Development and Implementation

11.8 Education

11.9 Tools

12 Conclusions

A Survey of Formal Methods Tools

B Survey of Formal Methods Applications

C Acronyms and Trademarks 